\title{
openheart Health literacy interventions for secondary prevention of coronary artery disease: a scoping review
}

\author{
Alison Beauchamp, ${ }^{1,2}$ Jason Talevski (D) ,1,2 Josef Niebauer, ${ }^{3,4}$ \\ Johanna Gutenberg, ${ }^{4,5}$ Emmanuel Kefalianos, ${ }^{6}$ Barbara Mayr, ${ }^{3,4}$ Mahdi Sareban, ${ }^{3,4}$ \\ Stefan Tino Kulnik (D) ${ }^{4,7}$
}

- Additional supplemental material is published online only. To view, please visit the journal online (http://dx.doi.org/10. 1136/openhrt-2021-001895).

To cite: Beauchamp A, Talevski J, Niebauer J, et al. Health literacy interventions for secondary prevention of coronary artery disease: a scoping review. Open Heart 2022;9:e001895. doi:10.1136/ openhrt-2021-001895

Received 8 November 2021 Accepted 5 January 2022
D Check for updates

(c) Author(s) (or their employer(s)) 2022. Re-use permitted under CC BY-NC. No commercial re-use. See rights and permissions. Published by BMJ.

For numbered affiliations see end of article.

Correspondence to Dr Alison Beauchamp; alison. beauchamp@monash.edu

\section{ABSTRACT}

Deficits in health literacy are common in patients with coronary artery disease (CAD), and this is associated with increased morbidity and mortality. In this scoping review, we sought to identify health literacy interventions that aimed to improve outcomes in patients with CAD, using a contemporary conceptual model that captures multiple aspects of health literacy. We searched electronic databases for studies published since 2010. Eligible were studies of interventions supporting patients with CAD to find, understand and use health information via one of the following: building social support for health; empowering people with lower health literacy; improving interaction between patients and the health system; improving health literacy capacities of clinicians or facilitating access to health services. Studies were assessed for methodological quality, and findings were analysed through qualitative synthesis. In total, 21 studies were included. Of these, 10 studies aimed to build social support for health; 6 of these were effective, including those involving partners or peers. Five studies targeted interaction between patients and the health system; four of these reported improved outcomes, including through use of teach-back. One study addressed health literacy capacities of clinicians through communication training, and two facilitated access to health services via structured follow-up-all reporting positive outcomes. Health literacy is a prerequisite for CAD patients to self-manage their health. Through use of a conceptual framework to describe health literacy interventions, we identified mechanisms by which patients can be supported to improve health outcomes. Our findings warrant integration of these interventions into routine clinical practice.

\section{INTRODUCTION}

Despite recent declines in mortality, coronary artery disease (CAD) remains a leading cause of death in adults. ${ }^{1} \mathrm{CAD}$ results in insufficient oxygen supply to the heart muscle and is the underlying cause of acute coronary syndrome (ACS), myocardial infarction (MI) and subsequent death. ${ }^{2}$ To attenuate the progression of CAD, secondary prevention guidelines for heart disease recommend ongoing management of cardiac risk factors through lifestyle changes, medication use and cardiac rehabilitation (CR). ${ }^{3}$ However, these behaviours can be difficult to initiate and sustain in the longer term, with studies finding adherence rates to secondary prevention medications of $35 \%-71 \%$ following $\mathrm{MI},{ }^{4}$ attendance rates of CR being approximately $30 \%{ }^{5}$ and smoking cessation rates 12 months post-MI at only $52 \% .^{6}$

One factor that may influence a patient's ability to initiate or maintain positive health behaviours is health literacy, which can be defined as 'the personal characteristics and social resources needed for individuals and communities to access, understand, appraise and use information and services to make decisions about health'. ${ }^{7}$ Up to $60 \%$ of cardiac patients have limited health literacy, ${ }^{89}$ which is associated with less engagement in healthy lifestyle behaviours, ${ }^{10}$ increased hospital readmissions ${ }^{11}$ and increased risk of mortality. ${ }^{11}$ However, little is known about effective interventions to address health literacy barriers among patients with CAD. A recent systematic review of health literacy interventions in various adult populations included just two studies in cardiovascular patients. ${ }^{12}$ This lack of evidence may be due to a strong focus on 'functional' health literacy within cardiac literature, that is, numeracy and literacy skills only. ${ }^{12-14}$ However, more recent interpretations of health literacy are broader, and encompass the resources and supports an individual needs to engage with and apply health information, and the role of clinicians and healthcare organisations in supporting this. ${ }^{15}$

Because health literacy is both an individual attribute and one which is impacted by the 'health literacy environment', ${ }^{15}$ interventions to address health literacy barriers must take account of a range of factors across the individual, their social networks and the healthcare system. ${ }^{13}$ A conceptual 


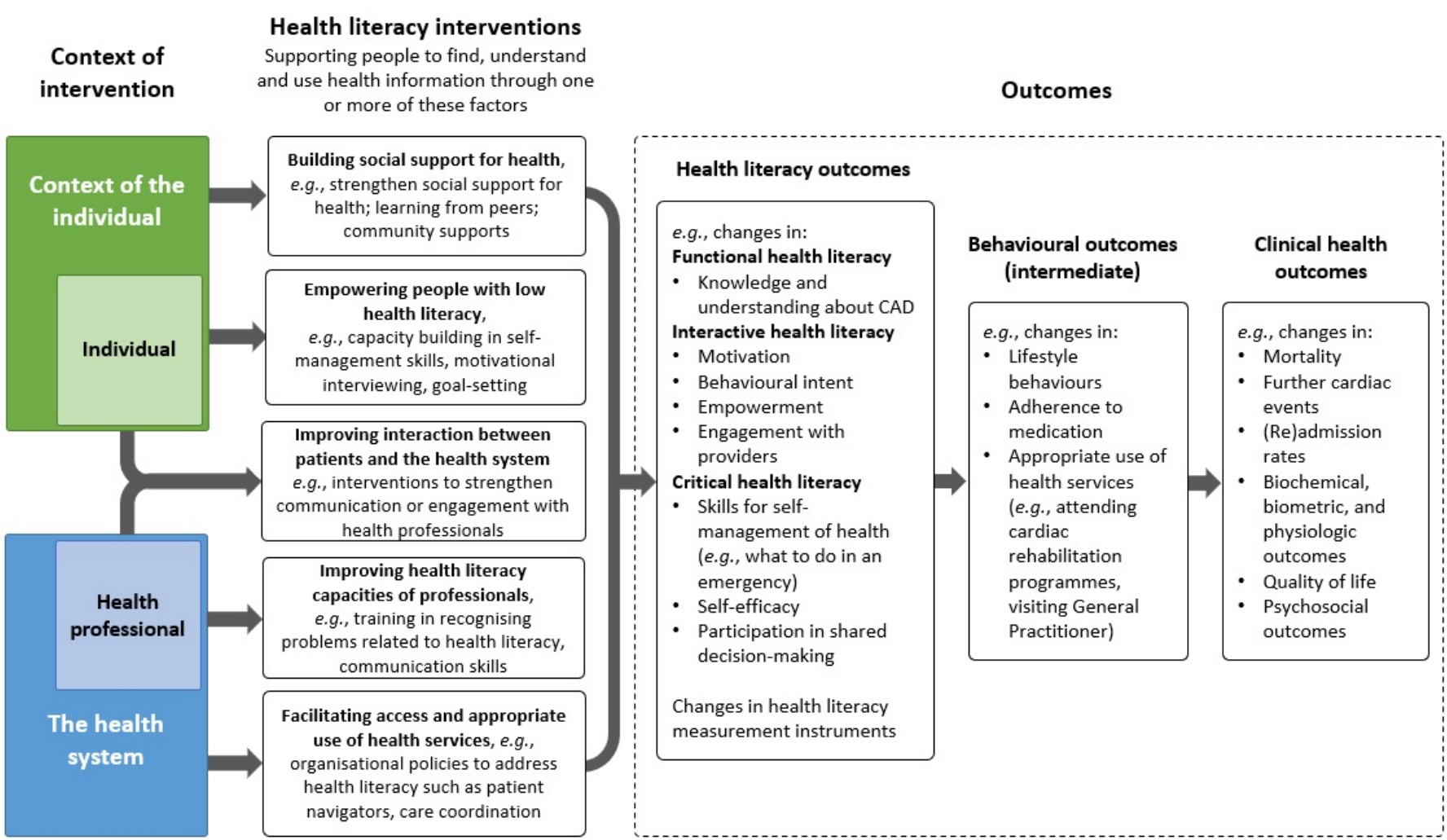

Figure 1 Health literacy intervention model for coronary artery disease (guided by the Geboers' Health Literacy Intervention Model).

model recently developed by Geboers ${ }^{16}$ considers that health literacy interventions should address at least one of the following factors: (1) the context of the individual (eg, family, peers); (2) empowerment of individuals with lower health literacy (eg, capacity building); (3) improving interactions between an individual and the healthcare system; (4) improving health literacy capacities of health professionals and (5) improving accessibility to health services. Accordingly, a 'health literacy intervention' can be defined as a programme or process that supports people to find, understand and use health information by targeting one or more of these five factors. Applying this conceptual model to patients with $\mathrm{CAD}$, intervention outcomes may be grouped into three interlinked categories: (1) health literacy outcomes, (2) behavioural outcomes and (3) clinical health outcomes (figure 1).

Given the limited evidence for effective interventions to address health literacy among cardiac patients, there is need for an up-to-date review based on broad search terms that capture the multidimensional nature of health literacy. The aim of this review was to describe the current evidence about health literacy interventions among patients with CAD using a conceptual model to guide our definition of a 'health literacy intervention'. Specific objectives were to identify the size and types of evidence, to map the evidence against the conceptual model and to describe any gaps in research.

\section{METHODS}

We conducted a scoping review to address our research objectives. Scoping reviews address an exploratory research question by mapping key concepts, types of evidence and gaps in research related to a defined area. ${ }^{17}$ This review was not registered in PROSPERO as protocols for scoping reviews are not accepted. A protocol for this review has been drafted and may be requested from the corresponding author.

\section{Step 1: identification of the research question}

The research question was 'Are health literacy interventions associated with improved health literacy, behavioural outcomes and/or clinical outcomes in CAD patients?'.

\section{Step 2: identification of relevant studies}

An electronic search using Ovid MEDLINE, CINAHL and PsycINFO was performed for literature published between January 2010 and February 2021 (online supplemental file 1). Reference lists from eligible studies, systematic reviews and grey literature were also reviewed. Inclusion criteria were defined according to the $\mathrm{Popu}-$ lation, Intervention, Comparison, Outcome, Study type framework (table 1). Additional inclusion criteria included peer-reviewed publications in English or German languages, accessible in full text, and published since 2010 (to capture more recent evidence). Studies of cardiac conditions other than CAD were excluded unless outcomes were reported separately for CAD. This 
Table 1 Study eligibility criteria according to the PICOS framework

Population The study recruited participants with established CAD (cardiovascular disease, coronary heart disease, acute MI, acute coronary syndrome), or if results were reported separately for participants with CAD when the study sample included other clinical groups.

$\begin{array}{ll}\text { Intervention(s) } & \begin{array}{l}\text { All interventions that fit the conceptual model of health literacy adopted for this review. The intervention was described as a 'health literacy } \\ \text { intervention' by the study authors; the authors examined whether the intervention was effective in people with low versus high health } \\ \text { literacy; or the intervention matched the health literacy definition of supporting people to find, understand and use information through } \\ \text { at least one of the mechanisms shown in the conceptual model in figure 1: building social support for health; empowering people with } \\ \text { lower health literacy; improving interaction between patients and the health system; improving the health literacy capacities of health } \\ \text { professionals; facilitating access and appropriate use of health services. }\end{array} \\ \text { Comparison } & \text { Any comparison, that is, an alternative intervention, usual care, or no care. } \\ \text { Outcome(s) } & \begin{array}{l}\text { Participant outcomes relating to changes in health literacy, health behaviours (eg, physical exercise, appropriate use of health services) or } \\ \text { clinical health outcomes (eg, hospital admissions, depression). }\end{array} \\ \text { Study types } & \begin{array}{l}\text { The study investigated the effect of an intervention, in a controlled (randomised, quasi-randomised or non-randomised) or uncontrolled (eg, } \\ \text { before and after comparison) study design. }\end{array}\end{array}$

CAD, coronary artery disease; MI, myocardial infarction; PICOS, Population, Intervention, Comparison, Outcome, Study.

covers studies of patients with heart failure. While heart failure is frequently a consequence of CAD, the aims of treatment may differ. We also excluded studies targeting primary CAD prevention.

\section{Step 3: study selection}

Title and abstracts were screened by the authors with ineligible records removed. Full texts of the remaining records were screened independently by two authors. Where a screening decision was uncertain, the final decision was made through discussion between $\mathrm{AB}$ and STK.

\section{Step 4: charting the data}

Data charting (extraction) from full-text articles was conducted by all reviewers. The following information was extracted: first author, publication year, country, study aim(s), study design, setting, sample description, intervention description, comparison/control group description (if applicable), outcome measures and key findings.

\section{Step 5: synthesis of results}

Findings were synthesised in a qualitative synthesis, using descriptive tables and concept maps to address the review objectives.

\section{Quality assessment}

The methodological quality of studies was assessed using the Effective Public Health Practice Project (EPHPP) Quality Assessment Tool, which has been developed and validated for use in controlled clinical trials, case-control and observational study designs. ${ }^{18}$ Criteria used to assess study quality were selection bias, study design, control for confounders, data collection methods and reporting of withdrawals and dropouts. Blinding was not used as a criterion as this was not relevant to most interventions. Studies were reported as weak (those with at least two out of five weak ratings), moderate (one weak rating) or strong (no weak ratings). Quality appraisal and data extraction were conducted by two independent reviewers, with differences resolved through discussion with a third reviewer.

\section{RESULTS}

\section{Study characteristics}

The electronic search yielded 2206 potential articles for review. From these, 21 unique studies were identified as eligible for inclusion (figure 2).

There were 14 randomised controlled trials (RCTs), 4 before and after studies, 2 prospective cohort studies and 1 controlled clinical trial. Most studies were from the USA $(n=4)$, Iran $(n=4)$ or China $(n=3)$; with two studies each from Canada, Denmark and the UK. Studies were implemented across various settings including hospitals $(n=12)$, outpatient clinics $(n=3)$, community health/ primary care centres $(n=3)$ and cardiac rehabilitation centres $(n=1)$. Most studies were in older adults (mean age: $>50$ years; $n=20)$ and included a higher percentage of men $(n=16)$. Studies were performed in subgroups of patients with CAD: four studies in patients with coronary heart disease (CHD); four among patients undergoing coronary artery bypass surgery (CABG); four in patients with ACS; three in patients with acute MI and one study in patients with angina pectoris. The methodological quality of included studies varied: 12 studies were rated as strong, 6 as moderate quality and 3 as weak. Study characteristics are summarised in table 2 .

\section{Intervention effectiveness}

Online supplemental file 2 provides a description of each health literacy intervention (grouped into the categories shown in figure 1) and summarises the key findings of each study. Although there was some variability among studies in relation to populations, settings and outcomes, 17 studies $(81 \%)$ reported positive findings for primary outcomes.

\section{Building social support for health}

Ten studies included an intervention that primarily focused on building social support for health. ${ }^{19-26}$ Three RCTs included interventions involving patients and partners and all achieved significant improvements in their primary outcome. ${ }^{19-21}$ Strategies included encouraging creation of a social support network or involving partners 


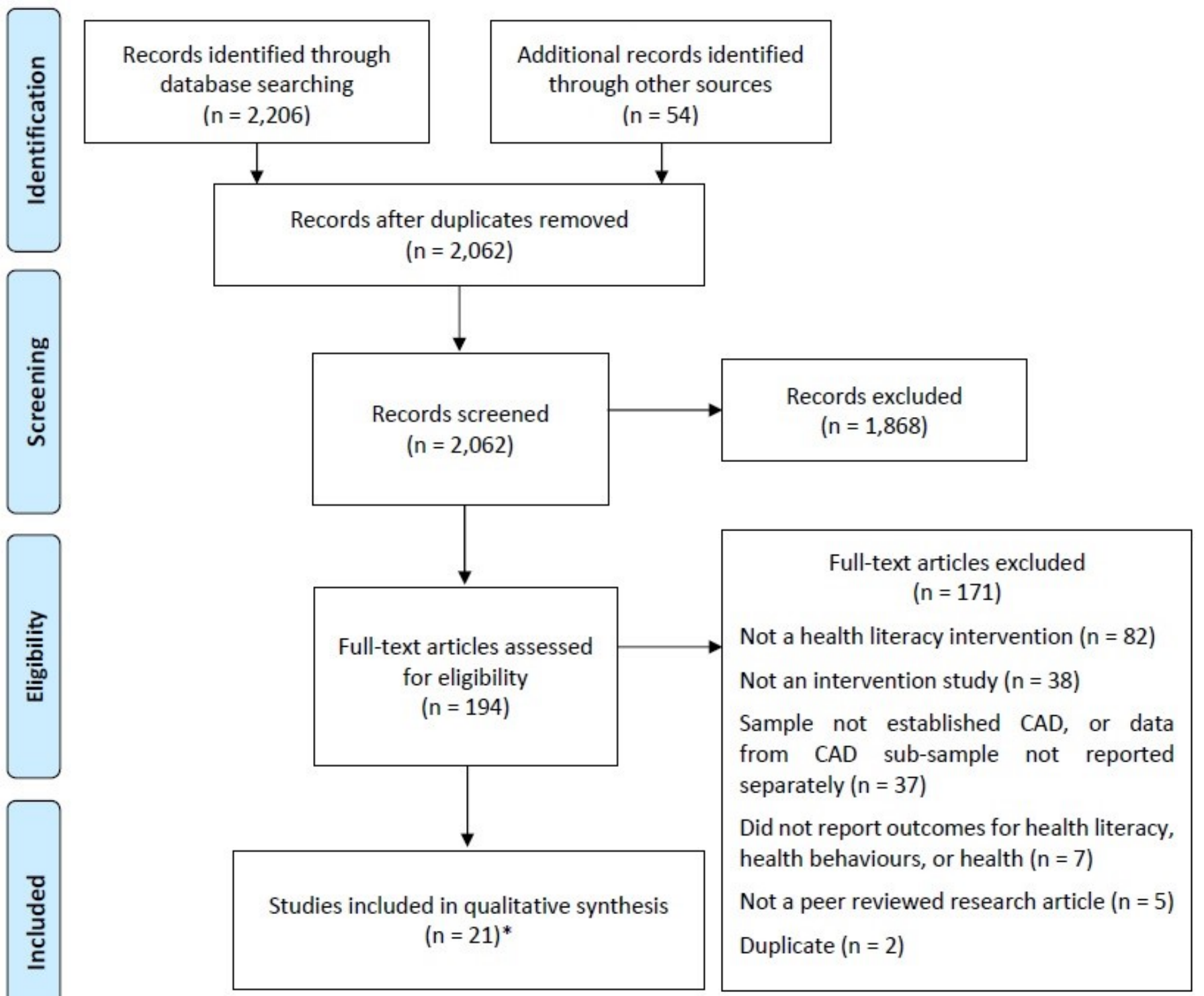

Figure 2 PRISMA flow diagram. *Two studies consisted of two publications reporting on different outcome measures and were recorded as one study in this review. CAD, coronary artery disease; PRISMA, Preferred Reporting Items for Systematic Reviews and Meta-Analysis.

in education. Of these RCTs, one reported increased physical activity and improved family support following an intervention in which patients with CHD and partners together developed action plans for home exercise. ${ }^{19}$ This theory-based intervention used a health behaviour change model called the Health Action Process Approach (HAPA). A fourth RCT also used the HAPA model to design an online patient education intervention. This RCT did not include partners and reported improvements in patient health behaviours but no effect on social support. ${ }^{27}$ Six studies assessed the use of peers for education or support. ${ }^{22-26} 28$ Overall findings were inconclusive, with four of the six studies showing improvements in primary outcomes. ${ }^{22} 232528$ These included one online intervention aiming to support women with $\mathrm{CAD}$ to learn about cardiac self-management from peers, ${ }^{28}$ and three face-to-face peer education sessions. ${ }^{22} 2325$ The two peerbased interventions that were less effective included one study in which post-CABG patients were matched with a peer volunteer who provided 6 weeks of telephone support $^{24}$ and an angina self-management programme facilitated by lay people with experience of CAD. ${ }^{26}$

Empowerment for people with low health literacy

This category refers to interventions that are targeted specifically at people with low health literacy. No studies were identified within this category.

Improving the interaction between patients and the health system/ health professionals

Five studies focused on improving interaction between patients and health professionals, with four of these reporting 

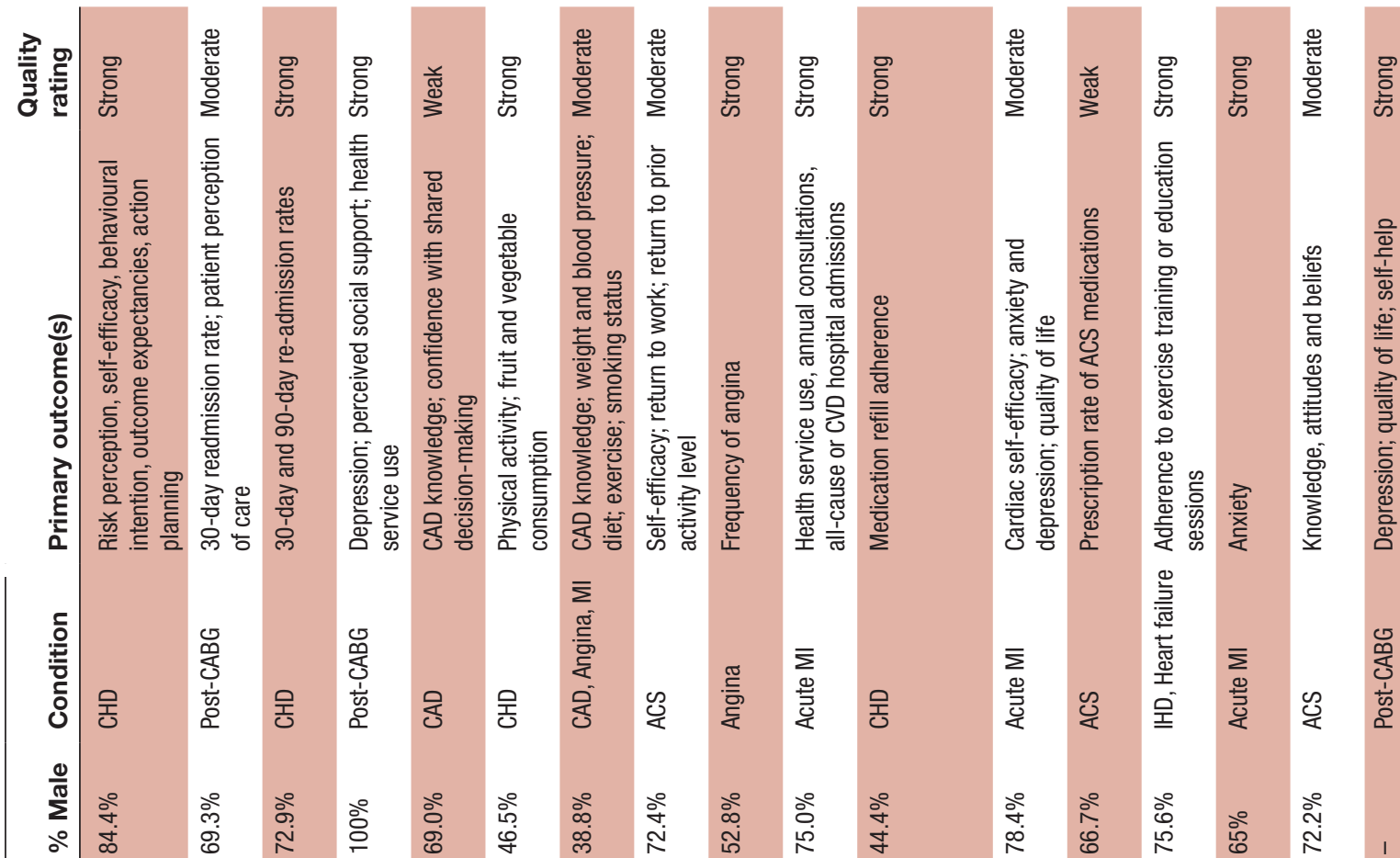

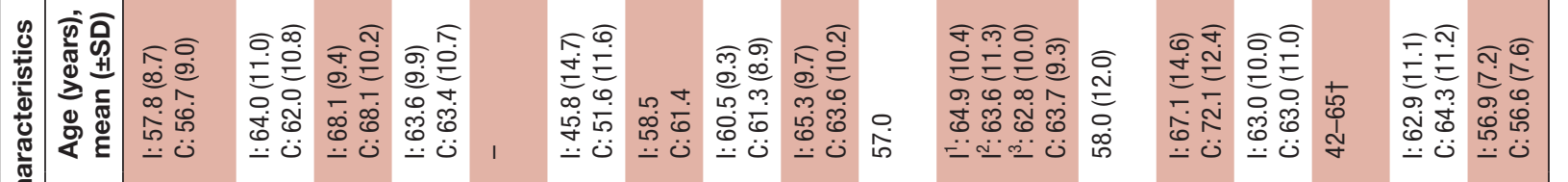

$\stackrel{\mathbb{N}}{\mathbb{N}}$

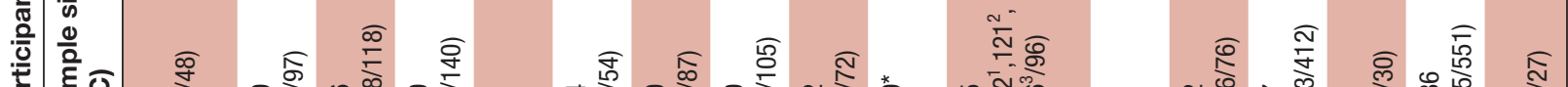
مी

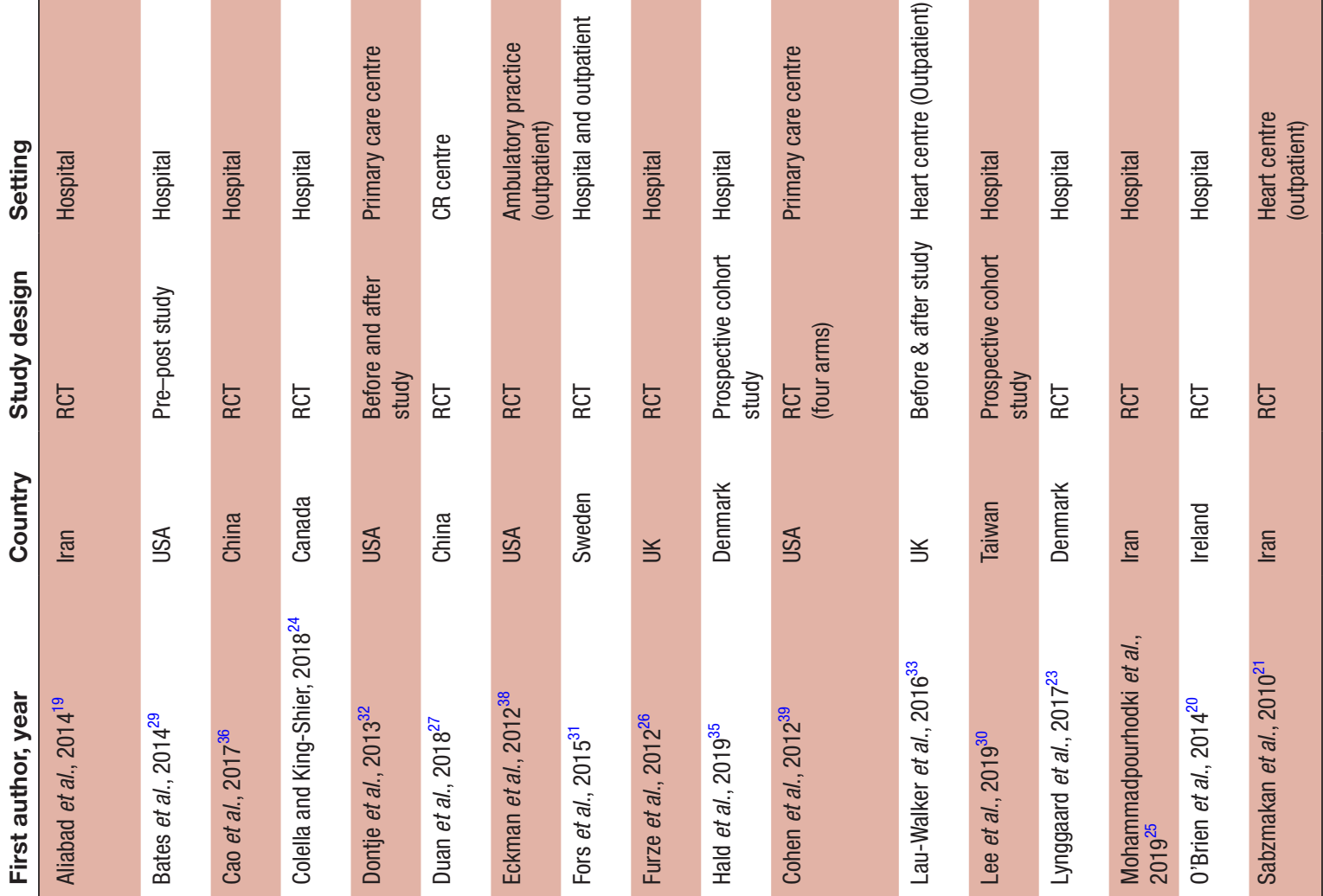




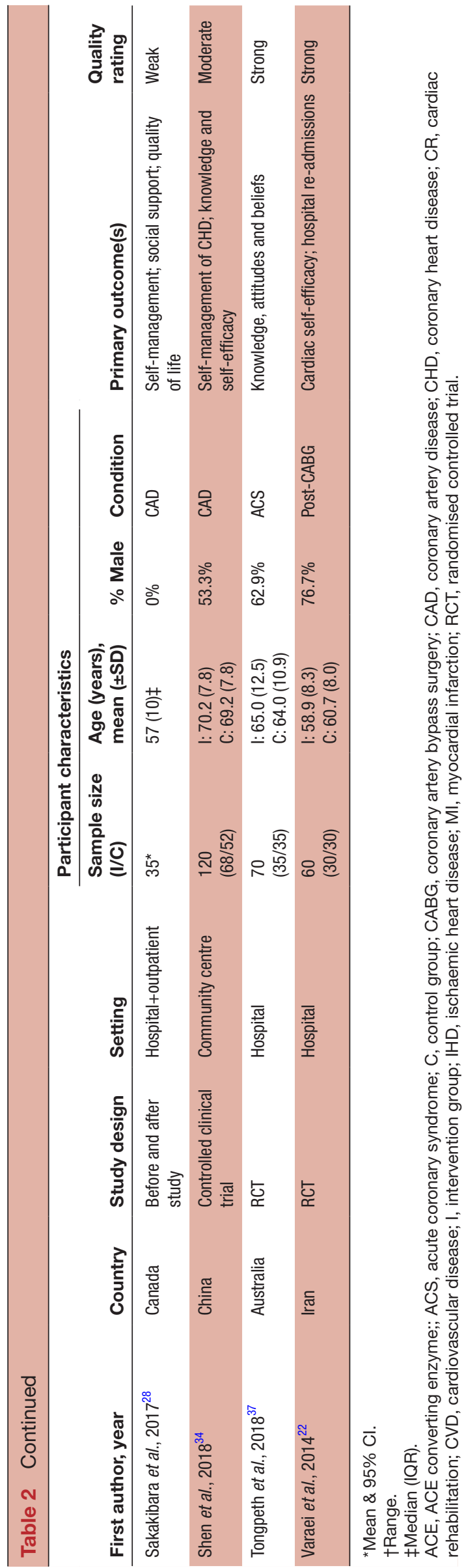

improved outcomes. ${ }^{29-32}$ In one before-and-after study, patients with CABG received education using 'teach-back' to check and clarify information. Intervention participants had significantly decreased readmission rates 30 -days postintervention. ${ }^{29}$ Two interventions aimed to increase frequency of interactions between patients and providers, both reporting positive findings in behavioural and health literacy outcomes. ${ }^{30} 31$ The two remaining studies aimed to build patients' capacity to communicate with health providers, including a beforeand-after study in patients with $\mathrm{CAD}^{32}$ and a pre-post study in patients with MI. ${ }^{33}$ This pre-post study ${ }^{33}$ was the least effective intervention in this category, with improvements seen only in single aspects of illness perception and quality of life.

\section{Building health literacy capacities of professionals}

Only one controlled clinical trial was identified in this category. This study described an education intervention for community nurses working with patients with CHD. ${ }^{34}$ The nurses' self-efficacy improved, as did selfmanagement behaviours among their patients.

\section{Facilitated access and appropriate use of health services}

Two studies included interventions that aimed to facilitate access and appropriate use of health services. ${ }^{3536} \mathrm{~A}$ prospective cohort study ${ }^{35}$ in socially vulnerable patients reported that receiving 'expanded CR' was associated with more annual chronic care consultations at 2 and 5 years. An RCT of a hospital-to-community transitional programme for patients with CHD (structured follow-up in the community; teach-back to reinforce patient understanding of treatment) found lower readmission rates in the intervention group. ${ }^{36}$

Described as a health literacy intervention or effects examined by health literacy level

No included studies were described explicitly as a 'health literacy intervention' by the study authors, although three studies examined intervention effects according to the health literacy levels of patients. ${ }^{37-39}$ All three studies reported that intervention effects did not differ between health literacy groups. Two studies showed significant improvements in their primary outcome, including an RCT evaluating an Avatar app for education of patients with CHD. ${ }^{37}$ This study found improved knowledge among the intervention group, but health literacy was not associated with these improved scores. In the second RCT, ${ }^{38}$ participants received printed booklets about $\mathrm{CAD}$ and watched videos of other patients who explained their own preferences. Improvements were seen in CAD knowledge and behaviours, with lower health literacy associated with a larger improvement. In a third $\mathrm{RCT}^{39}$ patients, received medication refill reminder postcards, illustrated medication schedules or both interventions for 1 year. No overall differences were seen across treatment groups, but this lack of effect did not differ according to patients' health literacy.

\section{DISCUSSION}

To our knowledge, this is the first scoping review appraising the broad range of health literacy 
interventions for patients with CAD. Most interventions had a primary focus on building social support for health or on improving the interaction between patients and the health system/health professionals. Key findings were: (1) involving partners in health education can be effective at reducing depression, increasing knowledge and improving physical activity; (2) use of peers for education or support may decrease anxiety, increase self-efficacy and improve health behaviours; (3) the teach-back method helps patients understand information and is associated with reduced hospital readmissions and (4) providing structured follow-up support may improve appropriate use of health services and reduce readmissions.

Evidence for the effectiveness of health literacy interventions among patients with $\mathrm{CAD}$ is limited in comparison to systematic reviews in other areas such as health promotion ${ }^{12}$ or chronic disease. ${ }^{12}{ }^{40}$ However, similar to CAD, much of this evidence focuses on functional health literacy only. Use of a conceptual framework in our study has enabled identification of a wider range of studies that go beyond functional health literacy and may be useful in other health conditions to identify characteristics of interventions associated with improved behavioural and clinical outcomes. In relation to types of evidence, two-thirds of the included studies were RCTs, and this is reflected in the relatively large proportion of studies of strong methodological quality.

We found no studies addressing empowerment of people with low health literacy, and a paucity of studies addressing health literacy capacities of health professionals or those facilitating access and appropriate use of health services. We also found heterogeneity between interventions, even within intervention categories, with regards to theoretical basis, recipients of the intervention, duration and frequency of interaction and target outcomes. Outcome measures were equally spread across the health literacy, behavioural and clinical health domains. However, few studies comprehensively covered more than one outcome aspect; therefore, it is difficult to conclude whether health literacy outcomes lead sequentially to behavioural and clinical health outcomes. This is a gap in the literature that limits our knowledge of the potential causal mechanisms and long-term health benefits of health literacy interventions.

Findings from this review suggest that involving partners is an effective way of building social support for health behaviours. ${ }^{19-21}$ Of note, two of these interventions used a theoretically informed educational approach (the HAPA model), including strategies for engaging family members. ${ }^{19} 21$ Other studies using this approach in non-CAD populations have also shown improved physical activity, ${ }^{41}$ and smoking cessation. ${ }^{42}$ For patients with $\mathrm{CAD}$, the practical and emotional help provided by others can assist with understanding health information and decision-making about management of their condition. ${ }^{43} 44$ This is aligned with the concept of 'distributed health literacy' where patients share tasks of finding, understanding and using health information with others, often drawing on health literacy abilities of others to manage their own health. ${ }^{44}$ Consideration should, therefore, be given to interventions that build social support systems to assist patients to find, understand and apply health information, including use of proven theoretically based approaches.

Improving partnerships between patients and health providers may also be effective, although of the five studies in this category, we found only two that focused on building patients' capacity to communicate with healthcare providers. ${ }^{32} 33$ This is an area for further investigation; for example, a 2017 systematic review found most evidence in healthcare communication is centred on improving the communication skills of providers rather than patients. ${ }^{45}$ We also found that interventions targeting both providers and patients were effective, including those using teach-back. ${ }^{29}$ The teach-back method of checking and clarifying information can be considered a health literacy intervention because it helps patients both understand information and recall it later at home. While there is strong evidence for the effectiveness of teach-back in chronic disease populations, ${ }^{46}$ surprisingly few papers have explored its role among patients with CAD.

We found few studies aiming to build health literacy capacity of health professionals or to empower patients with low health literacy. Some evidence from other settings indicates that interventions among lower health literacy groups are effective at increasing empowerment (eg, telehealth support ${ }^{47}$ community health education $)^{48}$ and these may warrant investigation in patients with CAD. This research should include vulnerable populations in whom health literacy and empowerment are known to be lower. ${ }^{1}$ Future studies in this area should also consider assessing health literacy using multidimensional tools rather than measuring functional health literacy only. ${ }^{14}$ Of note, those studies that did assess interventions across different health literacy groups found no difference in effects between patients with lower and higher health literacy; although all three used a functional health literacy instrument. ${ }^{37-39}$

It is noteworthy that only three studies in this review investigated interventions that incorporated digital technologies. Digital health approaches are progressing rapidly across all fields of medicine, including cardiology. The potential benefit of digital technologies to support heath literacy is manifold, such as equity of access to health-related information, convenient delivery of educational content in different audio-visual formats and languages and the possibility to incorporate user interaction for additional intervention effect. ${ }^{49}$ At the same time, digital technology interventions must consider the digital health literacy of user groups, that is, the degree to which individuals have the capacity to find, understand, appraise and apply health information from electronic sources. ${ }^{50}$ This review, therefore, highlights an opportunity for future research to develop and investigate digital health literacy interventions for patients with CAD. In 
line with key findings from this review, the effectiveness of these digital technologies may be further enhanced by strengthening social support or by targeting patientprovider partnerships.

\section{Strengths and limitations}

The strengths of this review include a rigorous methodology and comprehensive search strategy. Scoping reviews are an appropriate method to assess the size and scope of research literature and to identify the nature and extent of research evidence. ${ }^{17}$ Use of a conceptual framework enabled us to identify a wider range of studies that go beyond functional health literacy and can be used for future systematic reviews. Limitations should also be considered. While we attempted to include only those studies meeting our definition of a health literacy intervention, some studies have multiple mechanisms of effect, not all of which fit into our conceptual framework. As such, we cannot be clear that outcomes were due to the health literacy aspects of the intervention. Searches were limited to published studies, subjecting this review to the possibility of publication bias. Finally, the majority of included studies were conducted in high-income or middle-income countries, and our findings and conclusions may not be relevant for lower income countries where overall literacy of the population may be low, and health systems are poorly resourced. There is limited evidence describing health literacy in low-income countries, including in cardiac conditions. A recent systematic review ${ }^{51}$ identified that health literacy interventions in low-income and middle-income countries were primarily functional in nature, i.e., focused on improving knowledge and understanding at the individual level-there was less emphasis on the role of health services and systems in addressing health literacy barriers. Further research in low-income and middle-income countries is required that incorporates individual and system-level factors; however, it is important that this is locally driven with an understanding of contextual factors. ${ }^{52}$

\section{CONCLUSIONS}

Through the use of a broad conceptual health literacy framework, this scoping review has identified the available literature and described key characteristics of effective health literacy interventions for patients with CAD. These include social support by partners or peers, teachback, codesign of discharge plans, increased frequency of patient-provider interactions and facilitated access to health services. Our findings warrant integration of these interventions into clinical practice. Identified areas for future research are interventions for patients with CAD with low health literacy, health literacy skills of health professionals, facilitated access and appropriate use of health services, health literacy interventions, which employ digital technologies and interventions to improve digital health literacy.

\section{Author affiliations}

${ }^{1}$ Monash Rural Health, Monash University, Warragul, Victoria, Australia
${ }^{2}$ Department of Medicine-Western Health, The University of Melbourne, Melbourne, Victoria, Australia

${ }^{3}$ University Institute of Sports Medicine, Institute of Molecular Sports and Rehabilitation Medicine, Paracelsus Medical University, Salzburg, Austria ${ }^{4}$ Ludwig Boltzmann Institute for Digital Health and Prevention, Salzburg, Austria ${ }^{5}$ CAPHRI Department of Health Promotion, Maastricht University, Maastricht, The Netherlands

${ }^{6}$ Department of Physiotherapy, Western Health, Footscray, Victoria, Australia ${ }^{7}$ Faculty of Health, Social Care and Education, Kingston University and St George's, University of London, London, UK

Acknowledgements We wish to acknowledge the contribution of Dr Thomas Stütz to study selection and data extraction.

Contributors $A B$ conceived the study. $A B$ and STK designed the study. AB, STK and JT conducted literature searches. AB, STK, JG, EK, BM, MS and JT contributed to study selection and data extraction. AB, STK and JT conducted the quality assessments, summarised the results and drafted the manuscript. All authors critically revised the manuscript and approved the final version.

Funding This work was supported by the National Health \& Medical Research Council Grant Number: 1198006 to AB.

Competing interests None declared.

Patient consent for publication Not applicable.

Ethics approval This study does not involve human participants.

Provenance and peer review Not commissioned; internally peer reviewed.

Data availability statement Data sharing not applicable as no datasets generated and/or analysed for this study.

Open access This is an open access article distributed in accordance with the Creative Commons Attribution Non Commercial (CC BY-NC 4.0) license, which permits others to distribute, remix, adapt, build upon this work non-commercially, and license their derivative works on different terms, provided the original work is properly cited, appropriate credit is given, any changes made indicated, and the use is non-commercial. See: http://creativecommons.org/licenses/by-nc/4.0/.

\section{ORCID iDs}

Jason Talevski http://orcid.org/0000-0001-9180-8758

Stefan Tino Kulnik http://orcid.org/0000-0001-5419-6713

\section{REFERENCES}

1 Roth GA, Mensah GA, Johnson CO, et al. Global burden of cardiovascular diseases and risk factors, 1990-2019: update from the GBD 2019 study. J Am Coll Cardiol 2020;76:2982-3021.

2 Knuuti J, Wijns W, Saraste A, et al. 2019 ESC guidelines for the diagnosis and management of chronic coronary syndromes. Eur Heart J 2020;41:407-77.

3 Graham I, Atar D, Borch-Johnsen K, et al. European guidelines on cardiovascular disease prevention in clinical practice: Executive summary: fourth joint Task force of the European Society of cardiology and other societies on cardiovascular disease prevention in clinical practice (constituted by representatives of nine societies and by invited experts). Eur Heart J 2007;28:2375-414.

4 Greenland M, Knuiman MW, Hung J, et al. Cardioprotective medication adherence in Western Australians in the first year after myocardial infarction: restricted cubic spline analysis of adherenceoutcome relationships. Sci Rep 2020;10:4315.

5 Santiago de Araújo Pio C, Chaves GS, Davies P, et al. Interventions to promote patient utilisation of cardiac rehabilitation. Cochrane Database Syst Rev 2019;2:Cd007131.

6 Choi Y-J, Park J-S, Kim U, et al. Changes in smoking behavior and adherence to preventive guidelines among smokers after a heart attack. J Geriatr Cardiol 2013;10:146-50.

7 Dodson S. Health literacy toolkit for low and middle-income countries: a series of information sheets to empower communities and strenghten health systems. New Delhi: World Health Organization, 2015.

8 Machado B, Fernandes A, Cruzeiro S, et al. Cardiac rehabilitation program and health literacy levels: a cross-sectional, descriptive study. Nurs Health Sci 2019;21:406-12.

9 Diederichs C, Jordan S, Domanska O, et al. Health literacy in men and women with cardiovascular diseases and its association with the use of health care services - Results from the population- 
based GEDA2014/2015-EHIS survey in Germany. PLoS One 2018;13:e0208303.

10 Tschaftary A, Hess N, Hiltner S, et al. The association between sex, age and health literacy and the uptake of cardiovascular prevention: a cross-sectional analysis in a primary care setting. $J$ Public Health 2018;26:551-8.

11 Mayberry LS, Schildcrout JS, Wallston KA, et al. Health literacy and 1-year mortality: mechanisms of association in adults hospitalized for cardiovascular disease. Mayo Clin Proc 2018;93:1728-38.

12 Walters R, Leslie SJ, Polson R, et al. Establishing the efficacy of interventions to improve health literacy and health behaviours: a systematic review. BMC Public Health 2020;20:1040.

13 Stormacq C, Wosinski J, Boillat E, et al. Effects of health literacy interventions on health-related outcomes in socioeconomically disadvantaged adults living in the community: a systematic review. JBI Evid Synth 2020;18:1389-469.

14 Chan RWY, Kisa A. A scoping review of health literacy measurement tools in the context of cardiovascular health. Health Educ Behav 2019;46:677-88.

15 Batterham RW, Hawkins M, Collins PA, et al. Health literacy: applying current concepts to improve health services and reduce health inequalities. Public Health 2016;132:3-12.

16 Geboers B, Reijneveld SA, Koot JAR, et al. Moving towards a comprehensive approach for health literacy interventions: the development of a health literacy intervention model. Int J Environ Res Public Health 2018;15. doi:10.3390/ijerph15061268. [Epub ahead of print: 1506 2018].

17 Colquhoun $\mathrm{HL}$, Levac D, O'Brien KK, et al. Scoping reviews: time for clarity in definition, methods, and reporting. J Clin Epidemiol 2014;67:1291-4

18 Thomas BH, Ciliska D, Dobbins M, et al. A process for systematically reviewing the literature: providing the research evidence for public health nursing interventions. Worldviews Evid Based Nurs 2004:1:176-84.

19 Aliabad HO, Vafaeinasab M, Morowatisharifabad MA, et al. Maintenance of physical activity and exercise capacity after rehabilitation in coronary heart disease: a randomized controlled trial. Glob J Health Sci 2014;6:198-208

20 O'Brien F, McKee G, Mooney M, et al. Improving knowledge, attitudes and beliefs about acute coronary syndrome through an individualized educational intervention: a randomized controlled trial. Patient Educ Couns 2014:96:179-87.

21 Sabzmakan L, Hazavehei S, Morowatisharifabad M, Hasanzadeh M.:, et al. The effects of a PRECEDE-based educational program on depression, general health, and quality of life of coronary artery bypass grafting patients. Asian J Psychiatr 2010;3:79-83.

22 Varaei S, Shamsizadeh M, Cheraghi MA, Talebi M, et al. Effects of a peer education on cardiac self-efficacy and readmissions in patients undergoing coronary artery bypass graft surgery: a randomizedcontrolled trial. Nurs Crit Care 2017;22:19-28.

23 Lynggaard V, Nielsen CV, Zwisler A-D, VintherZwisler C, AnnDortheTaylor RS, et al. The patient education - Learning and Coping Strategies - improves adherence in cardiac rehabilitation (LCREHAB): A randomised controlled trial. Int J Cardiol 2017;236:65-70.

24 Colella TJ, King-Shier K. The effect of a peer support intervention on early recovery outcomes in men recovering from coronary bypass surgery: a randomized controlled trial. Eur $J$ Cardiovasc Nurs 2018;17:408-17.

25 Mohammadpourhodki R, Bagheri H, Basirinezhad MH, HoseinKeramati $\mathrm{M}$, et al. Evaluating the effect of lifestyle education based on peer model on anxiety in patients with acute myocardial infarction. J Complement Integr Med 2019;16. doi:10.1515/jcim2018-0132. [Epub ahead of print: 19 Jan 2019].

26 Furze G, Cox H, Morton V, Chuang V.:, et al. Randomized controlled trial of a lay-facilitated angina management programme. J Adv Nurs 2012;68:2267-79

27 Duan YP, Liang W, Guo L, JulianSi, Gang YanLippke S, et al. Evaluation of a web-based intervention for multiple health behavior changes in patients with coronary heart disease in home-based rehabilitation: pilot randomized controlled trial. J Med Internet Res 2018;20:e12052.

28 Sakakibara BM, Ross E, Arthur G, et al. Using Mobile-Health to connect women with cardiovascular disease and improve selfmanagement. Telemed J E Health 2017:23:233-9.

29 Bates OL, O'Connor N, Dunn D, et al. Applying STAAR interventions in incremental bundles: improving post-CABG surgical patient care. Worldviews Evid Based Nurs 2014;11:89-97.

30 Lee C-K, Lai C-L, Lee M-H, Fang-YingYeh T-S, et al. Reinforcement of patient education improved physicians' adherence to guideline- recommended medical therapy after acute coronary syndrome. PLoS One 2019;14:e0217444.

31 Fors A, Gyllensten H, Swedberg K, HannaSwedberg, KarlEkman $\mathrm{I}$, et al. Effectiveness of person-centred care after acute coronary syndrome in relation to educational level: subgroup analysis of a two-armed randomised controlled trial. Int $J$ Cardiol 2016:221:957-62.

32 Dontje K, Kelly-Blake K, Olomu A, Olomu K.:, Rothert A.:, et al. Nurse-Led group visits support shared decision making in stable coronary artery disease. J Cardiovasc Nurs 2013;28:269-76.

33 Lau-Walker M, Landy A, Murrells T. Personalised discharge care planning for postmyocardial infarction patients through the use of the Personalised Patient Education Protocol - implementing theory into practice. J Clin Nurs 2016;25:1292-300.

34 Shen Z, Jiang C, Chen L. Evaluation of a train-the-trainer program for stable coronary artery disease management in community settings: a pilot study. Patient Educ Couns 2018;101:256-65.

35 Hald K, Meillier LK, Nielsen KM, et al. Does socially differentiated cardiac rehabilitation affect the use of healthcare services after myocardial infarction? A 10-year follow-up study. BMJ Open 2019;9:e030807.

36 Cao X-Y, Tian L, Chen L, et al. Effects of a hospital-community partnership transitional program in patients with coronary heart disease in Chengdu, China: a randomized controlled trial. Jpn J Nurs Sci 2017;14:320-31.

37 Tongpeth J, Du HY, Clark RA. Development and feasibility testing of an avatar-based education application for patients with acute coronary syndrome. J Clin Nurs 2018;27:3561-71.

38 Eckman MH, Wise R, Leonard AC, Leonard R.:, A. C.: Dixon, et al. Impact of health literacy on outcomes and effectiveness of an educational intervention in patients with chronic diseases. Patient Educ Couns 2012;87:143-51.

39 Cohen MJ, Shaykevich S, Cawthon C, SunilPaasche-Orlow MK, et al. Predictors of medication adherence postdischarge: the impact of patient age, insurance status, and prior adherence. $J$ Hosp Med 2012;7:470-5.

40 Visscher BB, Steunenberg B, Heijmans M, et al. Evidence on the effectiveness of health literacy interventions in the EU: a systematic review. BMC Public Health 2018:18:1414.

41 Emdadi S, Hazavehie SMM, Soltanian A, et al. Predictive factors of regular physical activity among middle-aged women in the West of Iran, Hamadan: application of precede model. J Res Health Sci 2015;15:244-9.

42 Ochsner S, Luszczynska A, Stadler G, et al. The interplay of received social support and self-regulatory factors in smoking cessation. Psychol Health 2014;29:16-31.

43 Clayton C, Motley C, Sakakibara B. Enhancing social support among people with cardiovascular disease: a systematic scoping review. Curr Cardiol Rep 2019;21:123.

44 Edwards M, Wood F, Davies M, et al. 'Distributed health literacy': longitudinal qualitative analysis of the roles of health literacy mediators and social networks of people living with a long-term health condition. Health Expect 2015;18:1180-93

45 D'Agostino TA, Atkinson TM, Latella LE, et al. Promoting patient participation in healthcare interactions through communication skills training: a systematic review. Patient Educ Couns 2017;100:1247-57.

46 Talevski J, Wong Shee A, Rasmussen B, et al. Teach-back: a systematic review of implementation and impacts. PLoS One 2020;15:e0231350.

47 Long AF, Gambling T. Enhancing health literacy and behavioural change within a tele-care education and support intervention for people with type 2 diabetes. Health Expect 2012;15:267-82.

48 Lin S-C, Chen I-J, Yu W-R, et al. Effect of a community-based participatory health literacy program on health behaviors and health empowerment among community-dwelling older adults: a quasiexperimental study. Geriatr Nurs 2019;40:494-501.

49 Conard S. Best practices in digital health literacy. Int J Cardiol 2019;292:277-9.

50 Norman CD, Skinner HA. eHealth literacy: essential skills for consumer health in a Networked world. J Med Internet Res 2006;8:e9.

51 Meherali S, Punjani NS, Mevawala A. Health literacy interventions to improve health outcomes in low- and middle-income countries. HLRP: Health Literacy Research and Practice 2020;4:e251-66.

52 Talevski J, Sanders KM, Busija L, et al. Health service use and quality of life recovery 12 months following major osteoporotic fracture: latent class analyses of the International costs and utilities related to osteoporotic fractures study (ICUROS). J Bone Miner Res 2021;36:252-61. 